\title{
Trapped-ion quantum simulation of tunable-range Heisenberg chains
}

Tobias Graß ${ }^{1 *}$ and Maciej Lewenstein ${ }^{1,2}$

${ }^{\text {"Correspondence: }}$
tobias.grass@icfo.es
${ }^{1}$ ICFO-Institut de Ciències
Fotòniques, Av. Carl Friedrich Gauss,
3,08860 Castelldefels, Barcelona,
Spain
Full list of author information is
available at the end of the article

available at the end of the article

\begin{abstract}
Quantum-optical techniques allow for generating controllable spin-spin interactions between ions, making trapped ions an ideal quantum simulator of Heisenberg chains. A single parameter, the detuning of the Raman coupling, allows to switch between ferromagnetic and antiferromagnetic chains, and to modify the range of the interactions. On the antiferromagnetic side, the system can be tuned from an extreme long-range limit, in which any pair of ions interacts with almost equal strength, to interactions with a $1 / r^{3}$ decay. By exact diagonalization, we study how a system of up to 20 ions behaves upon tuning the interactions. We find that it undergoes a transition from a dimerized state with extremely short-ranged correlations towards a state with quasi long-range order, that is, algebraically decaying correlations. The dynamical evolution of the system after a local quench is shown to strongly vary in the two regimes: While in the dimerized limit, the excitation remains localized for long times, propagating spinons characterize the dynamics of the quasi-long-range ordered system. Taking a look onto the ferromagnetic side of the system, we demonstrate the feasibility of witnessing non-locality of quantum correlations by measuring two-particle correlators.
\end{abstract}

Keywords: quantum simulations with trapped ions; spin models

\section{Introduction}

A paradigm system of quantum mechanics which may exhibit intriguing quantum properties like entanglement and non-locality are two spins. By increasing the number of spins, more complex behavior may emerge. In fact, a large variety of condensed matter phenomena, ranging from metal-insulator transition to superfluidity or superconductivity, are successfully described by mapping the relevant low-energy Hilbert space onto a spin model [1]. Moreover, spin models may describe spin-liquid phases which exhibit topological order. In recent years, technological progress in manipulating atoms on the quantum level has allowed to explicitly engineer spin models [2]. This has opened the opportunity for testing the foundations of quantum mechanics, for simulating complex many-body behavior, and for studying the dynamics of interacting spin chains [3-5].

A very promising quantum simulator are trapped ions, with widely spread applications ranging from relativistic quantum mechanics [6,7], topological systems [8], fermionic lattice models [9], to neural networks [10]. Trapped ions can be prepared in such a way that their dynamics is mainly restricted to some internal states of the ions, while the external motion is cooled down to only a few phonons. The internal states then represent a

C) 2014 Graß and Lewenstein; licensee Springer on behalf of EPJ. This is an Open Access article distributed under the terms of the Creative Commons Attribution License (http://creativecommons.org/licenses/by/2.0), which permits unrestricted use, distribution, and reproduction in any medium, provided the original work is properly cited. 
(pseudo)spin, and phonon-mediated spin-spin interactions can be implemented [11, 12]. This has already led to the experimental realization of SU(2) Ising models in one and two spatial dimensions [13-16], and, very recently, to the experimental study of entanglement dynamics in Ising and XY chains $[17,18]$. The implementation of more complicated spin models has been suggested, e.g. of Heisenberg and XY models [12, 19], or models with higher spin [20]. Furthermore, the tunability of the phonon-mediated interaction allows to study models with long-range interactions. Roughly, interactions with $1 / r^{\alpha}$ decay have been engineered for $0 \lesssim \alpha \lesssim 3[16]$.

This flexibility suggests an implementation of tunable-range spin models in trapped ions. While both theoretical and experimental literature so far has focussed on Ising- or XY-type quantum simulations [13-19, 21], here we consider a trapped-ion implementation of the Heisenberg model. In particular, by assuming an experimental setup as sketched in Figure 1(a), (b), we study the influence of a single control parameter on the quantum simulation, the detuning of the Raman coupling. As shown in Figure 1(c), (d), this parameter controls the range of the interactions. Modifying it may bring our quantum simulation close to different antiferromagnetic variants of the Heisenberg model: the HaldaneShastry model [22-24], the Majumdar-Ghosh model [25], or the Lipkin-Meshkov-Glick model [26]. While the Haldane-Shastry model describes antiferromagnetic order with al-

(a) Level scheme.

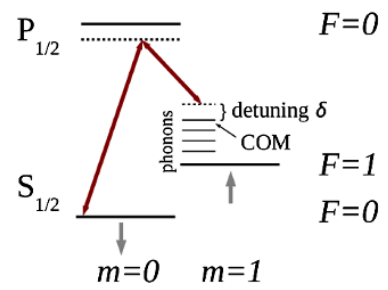

(c) Interactions for $N=20$ ions.

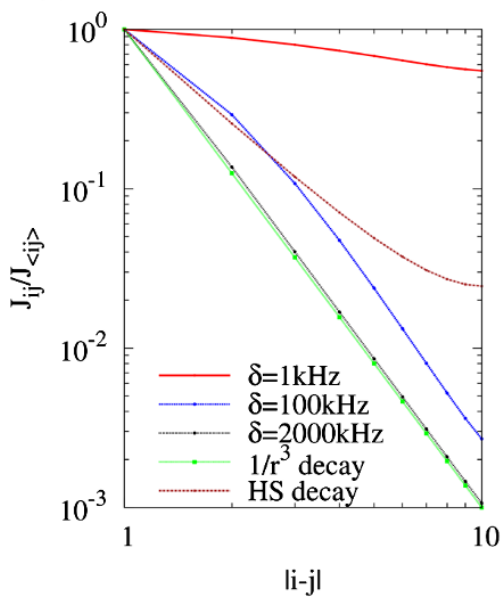

(b) Experimental setup.

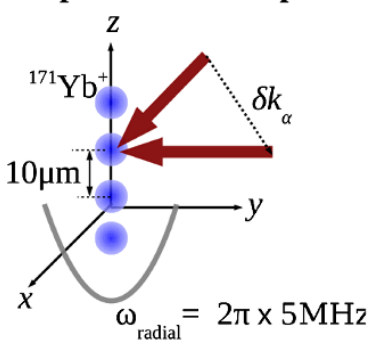

(d) For $N=200$ ions.

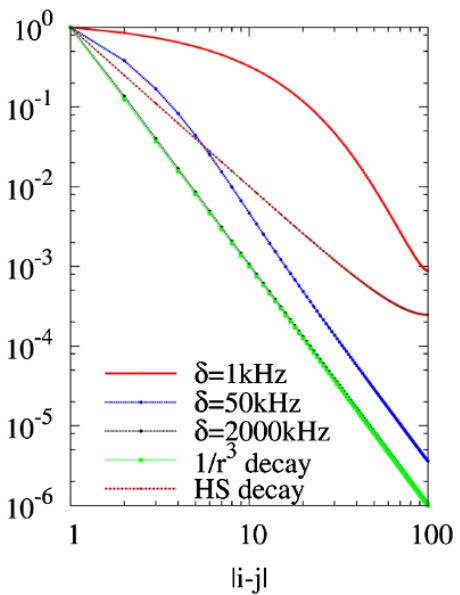

Figure 1 Engineering spin-spin interactions. (a), (b) Level scheme and setup for a possible implementation of spin-spin interactions in ${ }^{171} \mathrm{Yb}^{+}$. (c), (d) Interaction strengths $J_{i j}$ between one ion in the center and the other ions, for (c) $N=20$ or (d) $N=200$, and different detunings from the center-of-mass (COM) mode. We have used the parameters specified in (b). Interactions are compared with the interactions of the Haldane-Shastry (HS) model (brown lines), and with $1 / r^{3}$ interactions (green lines). 
gebraic decay of correlations, the Majumdar-Ghosh model provides a parent Hamiltonian for a fully dimerized ground state, that is a ground state with extremely short-range correlations. In the Lipkin-Meshkov-Glick model, spin-spin interactions are independent from distance, and the system is thus described in terms of global spin operators.

In this paper, we first provide an overview of the different models in Section 2. We then discuss the ionic setup in Section 3. In Section 4, we show that by increasing the range of interactions, a transition from a Haldane-Shastry-like quasi-long-range order to a Majumdar-Ghosh-like dimerized order can be observed in the ionic system. In Section 5, we apply different quenches to the system, and study the subsequenct dynamical evolution of the system. We consider local quenches, e.g. by a sudden application of a magnetic field to a single ion, and show that the dynamics in the dimerized regime differs significantly from the one in a system with quasi-long-range order. While in the former case the excitations remain localized for a long time, in the latter they spread in good agreement with the velocity obtained analytically for the Haldane-Shastry model. For global quenches, realized by apruptly changing the range of interactions and thereby sweeping through the dimerization transition, we study the correlation spreading, which is found at an increased velocity. In Section 6, we consider the Lipkin-Meshkov-Glick limit on the ferromagnetic side, which has been suggested for witnessing non-locality of quantum correlations [27]. Finally, we provide a summary in Section 7. Moreover, our paper contains two appendices: In Appendix 1, we provide details to the ionic setup, and in Appendix 2, we discuss the relation between the ionic model and a spin model.

\section{The models}

The Hamiltonian of a Heisenberg chain of $N$ spin-1/2 particles generally reads

$$
H=\sum_{i, j}^{N} \sum_{\alpha=x, y, z} J^{(i, j)} S_{\alpha}^{(i)} S_{\alpha}^{(j)},
$$

where $S_{\alpha}^{(i)}=\frac{\hbar}{2} \sigma_{\alpha}^{(i)}$, and $\sigma_{\alpha}^{(i)}$ denotes a Pauli matrix for the spin at position $i$. In this paper we focus on the antiferromagnetic side of this model, that is, the model with interaction strengths $J^{(i, j)}>0$.

The functional behavior of $J^{(i, j)}$ as $|i-j|$ may crucially influence the physics of the model. Let us in the following discuss the different cases which are important for our application.

\subsection{Haldane-Shastry model}

The Haldane-Shastry model is paradigmatic for Heisenberg chains with antiferromagnetic long-range interactions. It stands out through its analytical solution in terms of a Gutzwiller ansatz $[22,23]$. The model is characterized by interactions which decay quadratically with the distance $d_{i j}$ between the two spins, that is, $J_{\alpha}^{(i, j)} \equiv J d_{i j}^{-2}$. Periodic boundary conditions are imposed by arranging the spins on the unit circle, that is, with positions $z_{k}=\exp \left[\frac{2 \pi i}{N} k\right]$. In this case, $d_{i j}=2 \sin \left(\frac{\pi}{N}|i-j|\right)$. For a chain with even number of spins, the ground state reads

$$
\left|\Psi_{0}\right\rangle=\sum_{\left\{z_{1}, \ldots, z_{M}\right\}} \Psi_{0}\left(z_{1}, \ldots, z_{M}\right) S_{z_{1}}^{+} \cdots S_{z_{M}}^{+}|\downarrow \downarrow \cdots \downarrow\rangle,
$$


with $M=N / 2, S_{z_{i}}^{+}=|\uparrow\rangle\left\langle\left.\downarrow\right|_{z_{i}}\right.$ a raising operator of the spin at position $z_{i}$, and the coefficients of each Fock state given by the wave function

$$
\Psi_{0}\left(z_{1}, \ldots, z_{M}\right)=\prod_{i<j}^{M}\left(z_{i}-z_{j}\right)^{2} \prod_{i=1}^{M} z_{i} .
$$

This function has a remarkable similarity to the Laughlin wave function for two-dimensional systems in the fractional quantum Hall regime [28]. The similarities can be extended to the excited states of the model which are spinons with certain anyonic properties: Obtained as a superposition of spin flips, excitations live in an integer-spin Hilbert space, but, only occuring pairwise, each spinon carries half-integer spin. In that sense, the spinon represents a quasiparticle with a fractional quantum number. Similarly to excitations in Laughlin's state, the spinon wavefunctions can be obtained from the ground state by formally piercing a hole localized to position $\eta$ into it:

$$
\Psi_{\eta}\left(z_{1}, \ldots, z_{M}\right)=\prod_{i}\left(\eta-z_{i}\right) \Psi_{0}\left(z_{1}, \ldots, z_{M}\right) .
$$

Assuming now an odd number of spins, the state of a $\downarrow$-spinon localized at $\eta$ is given by $\left|\Psi_{\eta \downarrow}\right\rangle=\sum_{\left\{z_{1}, \ldots, z_{M}\right\}} \Psi_{\eta}\left(z_{1}, \ldots, z_{M}\right) S_{z_{1}}^{+} \cdots S_{z_{M}}^{+}|\downarrow \downarrow \cdots \downarrow\rangle$, where $M=(N-1) / 2$. By Fourier transformation of the localized spinon wave function, one obtains the wave function for a propagating spinon, which is an the exact solution of the model:

$$
\Psi_{p_{m}}\left(z_{1}, \ldots, z_{M}\right)=\sum_{\eta}\left(\eta^{*}\right)^{m} \Psi_{\eta}\left(z_{1}, \ldots, z_{M}\right)
$$

where $m$ is an integer defining the momentum $p_{m}=-(2 \pi / N) m+\pi(1-1 / 2 N)$. Evaluating the energies of $\Psi_{0}$ and $\Psi_{p_{m}}$, one obtains the spinon excitation energy as [24]:

$$
\epsilon\left(p_{m}\right)=\frac{J N^{2}}{4 \pi^{2}}\left[\frac{p_{m}}{2}\left(\pi-p_{m}\right)+\frac{\pi^{2}}{8 N^{2}}\right]
$$

From this, we read off the spinon velocity $v_{s}=\frac{J N^{2}}{8 \pi}$. We will later compare this result with the spinon velocity in an open chain. Therefore we have to note that the distance of neighboring spins on the unit circle is $2 \pi / N$, while on the open chain we conveniently take it as a unit of length. Accordingly, one has to rescale $J \rightarrow J \frac{4 \pi^{2}}{N^{2}}$, and the spinon velocity reads

$$
v_{s}=J \frac{\pi}{2}
$$

Returning to the ground state properties of the Haldane-Shastry model, we shall consider its spin correlations. It has been shown analytically that the model supports correlations with a power-law decay [29]

$$
\left\langle\sigma_{z}^{(i)} \sigma_{z}^{(j)}\right\rangle \propto \frac{(-1)^{|i-j|}}{|i-j|}
$$

With this criterion, the Haldane-Shastry model, despite the long-range interactions, supports the same quantum phase as the Heisenberg chain with nearest-neighbor interac- 
tions, also characterized by quasi long-range spin order. In the next subsection, we will introduce a model which is in contrast to this behavior.

\subsection{Majumdar-Ghosh model $/ J_{1}-J_{2}$ model}

The Heisenberg model with nearest-neighbor and next-nearest-neighbor interactions, $J_{1}$ and $J_{2}$, is called $J_{1}-J_{2}$-model. It is known to exhibit a dimerization transition when $J_{2} / J_{1} \gtrsim 1 / 4$ [30-32]. The physical consequences of the dimerization become clearest for $J_{2}=0.5 J_{1}$ and periodic boundary conditions. Then the model becomes identical to the Majumdar-Ghosh model [25], which is solved by two degenerate ground states $\left|\Psi_{+}\right\rangle$and $\left|\Psi_{-}\right\rangle$. These states are obtained by bringing every second nearest-neighbor pair into a spin singlet configuration, such that the total state is a product over singlet bonds:

$$
\left|\Psi_{ \pm}\right\rangle=\prod_{i=1}^{N / 2}\left(|\uparrow \downarrow\rangle_{2 i, 2 i \pm 1}-|\downarrow \uparrow\rangle_{2 i, 2 i \pm 1}\right) / \sqrt{2}
$$

It is obvious that in such dimer state, any spin is fully correlated to one nearest neighbor, but fully uncorrelated with the other spins. In other words, no long-range spin correlations exist. Note that for a system with open boundary conditions, the spin at position 1 and the spin at position $N$ do not interact, and therefore the ground state is uniquely given by $\left|\Psi_{-}\right\rangle$.

\subsection{Lipkin-Meshkov-Glick model}

By strengthening interactions between distant spins, one approaches the Lipkin-MeshkovGlick model [26] in which interactions are spatially independent, that is, $J^{(i, j)}=J$. With this, the Hamiltonian is rewritten as $H=J\left(\mathbf{S}^{2}-\frac{3}{4} N\right)$ with the total spin operator $\mathbf{S}=$ $\sum_{i}\left(\sigma_{x}^{(i)}, \sigma_{y}^{(i)}, \sigma_{z}^{(i)}\right)$. For antiferromagnetic interactions, any singlet state is thus a ground state, leading to a huge degeneracy. The number of singlet states formed by $N$ spin-1/2 particles is given by the Catalan number

$$
C_{n}=\frac{N !}{\left(\frac{N}{2}\right) !\left(\frac{N}{2}+1\right) !} \text {. }
$$

However, this degeneracy is lifted by any small spatial dependence of the interactions.

As we will see below, the ionic systems approaches the Lipkin-Meshkov-Glick limit when the Raman coupling is close to resonance with the center-of-mass phononic mode. By going through the resonance, one is able to swap the sign of the interactions, thus both the antiferromagnetic and the ferromagnetic Lipkin-Meshkov-Glick model can be realized. On the ferromagnetic side, the ground states are the symmetric Dicke states, that is, symmetric superpositions of all states with a fixed spin polarization, that is with a fixed number $N_{\uparrow}\left(N_{\downarrow}\right)$ of $\uparrow-(\downarrow-)$ spins. The unnormalized, symmetric Dicke states read

$$
\left|D_{N_{\uparrow}, N_{\downarrow}}\right\rangle \equiv \sum_{\left\{i_{1}, \ldots, i_{N}\right\}}|\uparrow\rangle_{i_{1}} \cdots|\uparrow\rangle_{i_{N_{\uparrow}}}|\downarrow\rangle_{i_{N_{\uparrow}+1}} \cdots|\downarrow\rangle_{i_{N_{\uparrow}+N_{\downarrow}}}
$$

Apparently, the ground state degeneracy is $(N+1)$-fold, and, as each Dicke state has a different spin polarization, it can be lifted by a polarizing field term $\sim h \sum_{i} \sigma_{z}^{(i)}$. While such term will make the fully polarized state $|\downarrow \cdots \downarrow\rangle$ the unique ground state, one can 
also obtain the polarized Dicke state $\left|D_{N / 2, N / 2}\right\rangle$ as the unique ground state by reducing the Heisenberg XXX interactions to XX interactions. The Hamiltonian then reads

$$
H_{\mathrm{LMG}}=J \sum_{i<j}\left(S_{x}^{(i)} S_{x}^{(j)}+S_{y}^{(i)} S_{y}^{(j)}\right)+h \sum_{i} S_{z}^{(i)}
$$

with $\left|D_{N / 2, N / 2}\right\rangle$ the unique ground state for $J<0$ and $h=0$.

\section{The ionic system}

Tunable-range Ising models have already been implemnted in trapped ions [13, 14]. In these experiments, two-levels ions are confined to a line by a Paul trap, and their motional state is cooled to only a few phonons. Strong spin-spin interactions are then achieved by applying state-dependent forces on the ions. In Ref. [13], Ising-type interactions of the form $\sigma_{z}^{(i)} \sigma_{z}^{(j)}$ are generated, whereas Ref. [14] describes the production of interactions of the form $\sigma_{x}^{(i)} \sigma_{x}^{(j)}$ or $\sigma_{y}^{(i)} \sigma_{y}^{(j)}$. As reviewed in Ref. [33], both approaches have basically the same footing, and it is possible to combine them. Instead of an Ising coupling, one then obtains, in the first place, an XYZ-model. By making all interactions equal, one gets the Heisenberg model.

An important difference between the ionic system and the ideal models discussed above are the boundary conditions: For the most feasible experimental implementation, they are open, while periodic boundary conditions are convenient for a theoretical description. In general, the effect of boundary conditions is minimized by scaling up the system. For systems of up to $N=20$ ions, we will in the following discuss how close the connection to the Haldane-Shastry model and the Majumdar-Ghosh model can be made by tuning the range of the interaction.

To this goal, let us first briefly review how the desired spin-spin interactions can be engineered. For each coupling, $\sum_{i<j} \sigma_{\alpha}^{(i)} \sigma_{\alpha}^{(j)}$, a pair of Raman lases is set up, as depicted in Figure 1(a), (b). Making several assumptions, which are sketched in the Appendix 1 and detailed in Ref. [33], the Hamiltonian for the interaction of the ions with the photons is given by $H(t)=\sum_{\alpha=x, y, z} h_{\alpha}(t)$, with

$$
h_{\alpha}(t)=\frac{\hbar \Omega_{\alpha}}{2} \sum_{i=1}^{N} \sum_{m} \eta_{m}\left(\hat{a}_{m} \mathrm{e}^{-i\left(\omega_{\alpha}-\omega_{0}-\omega_{m}\right) t}+\text { H.c. }\right) \sigma_{\alpha}^{(i)},
$$

for $\alpha=\mathrm{x}, \mathrm{y}$, and

$$
h_{z}(t)=\frac{i \hbar \Omega_{z}}{2} \sum_{i=1}^{N} \sum_{m} \eta_{m}\left(\hat{a}_{m} \mathrm{e}^{-i\left(\omega_{z}-\omega_{m}\right) t}+\text { H.c. }\right) \sigma_{z}^{(i)} .
$$

Here, $\hbar \omega_{0}$ is the energy difference between the two internal levels, $\hat{a}_{m}$ is the annihilation operator for phonons denoted by $m$ and with frequency $\omega_{m}$. The Rabi frequencies of the couplings are denoted by $\Omega_{\alpha}$, and $\omega_{\alpha}$ are the frequencies of the fields. Furthermore, the strength of each coupling depends on the Lamb-Dicke paremeters $\eta_{m}$, which are explicitly defined in the Appendix 1. For all couplings $\alpha$, the wave vector difference of the photons, $\delta \mathbf{k}_{\alpha}$, is assumed to be transverse to the ion chain, so the sum over $m$ reduces to a sum over $N$ transverse modes. 
It has been shown in Ref. [14], for a system with a single coupling term $h_{\alpha}$, that the time evolution can be made identical to the one of a spin system with the Hamiltonian

$$
H_{\alpha}=\frac{1}{\hbar} \sum_{i \leq j} J_{\alpha}^{(i, j)} S_{\alpha}^{(i)} S_{\alpha}^{(j)}
$$

where the spin-spin interaction strength is given by

$$
J_{\alpha}^{(i, j)}=\Omega_{\alpha}^{2} \sum_{m} \frac{\eta_{m \alpha}^{(i)} \eta_{m \alpha}^{(j)}}{\tilde{\omega}_{\alpha}-\omega_{m}} .
$$

Here, $\tilde{\omega}_{\alpha} \equiv \omega_{\alpha}-\omega_{0}$ for $\alpha=\mathrm{x}, \mathrm{y}$, whereas $\tilde{\omega}_{\alpha} \equiv \omega_{\alpha}$ for $\alpha=\mathrm{z}$. Some details of the derivation of this formula are provided in the Appendix 2. In particular, we generalize to the case of more than one coupling, and show that the couplings can be chosen such that they do not interfere. The Hamiltonian then is effectively given by

$$
H=\frac{1}{\hbar} \sum_{\alpha} \sum_{i \leq j} J_{\alpha}^{(i, j)} S_{\alpha}^{(i)} S_{\alpha}^{(j)}
$$

with all $J_{\alpha}^{(i, j)}$ given by Eq. (16). These interactions can be tuned by varying the frequency of the Raman laser. Choosing it close to the frequency of the center-of-mass mode, the strength of the induced interactions will barely depend on the particles' position, $J^{(i, j)} \approx J$. The sign of $J$ can be made positive (negative) by tuning above (below) the center-of-mass frequency. Close to resonance, the system is similar to the Lipkin-Meshkov-Glick model. Due to the strong nearest-neighbor and next-nearest-neighbor interactions this limit is somewhat similar to the dimerized $J_{1}-J_{2}$-model.

By increasing the detuning from the center-of-mass mode, $J^{(i, j)}$ is made spatially dependent, and in the limit of large detuning, a $1 / r^{3}$ decay can be achieved. For intermediate values of the detuning, the interactions may approximate a quadratic decay for sufficiently small $r$, as shown in Figure 1(c), (d). The most dominant interactions then agree quantitatively well with the interactions of the Haldane-Shastry model. Further techniques could be applied in order to achieve a better agreement of the ion setup with a particular model, e.g. by individually addressing of the ions [34]. However, here we restrict ourselves to the simplest implementation which already exhibits rich physics.

The feasibility of the experimental implementation depends on different factors which potentially reduce the fidelity. In the first place, one should note that the effective Hamiltonian (17) has been obtained by neglecting a residual spin-phonon coupling. This implies an error $\epsilon_{s p} \approx 4(1+\bar{n}) \eta^{2}$, with $\bar{n}$ the mean phonon number [12]. This error is small in the Lamb-Dicke regime, $\eta \ll 1$, typically of the order of few percents [15]. Another source of error, which becomes particularly important for small detunings, are Raman-induced phonon excitations, with a probability given by $p_{\mathrm{ph}}=(\eta \Omega / \delta)^{2} \sim J / \delta$. Keeping this error small at a given detuning therefore sets an upper limit to the coupling parameter $J$. On the other hand, $J$ should be sufficiently large compared to imperfections like field imhomogenities or spontanous emission rates. The recent experiments in Maryland [17] and in Innsbruck [18] with up to 15 trapped ions operate at a $J_{\max } \sim 400 \mathrm{~Hz}$, much larger than 
the estimated inhomogenities $<20 \mathrm{~Hz}$ [18]. We will find in the next section that dimerization occurs at a detuning of the order of $10 \mathrm{kHz}$, that is, slightly below the lowest detuning realized in Ref. [18]. In this regime, photon errors are estimated to be $p_{\mathrm{ph}}=0.04$.

An important part of trapped-ion quantum simulations are the well developped readout techniques. In particular, it is possible with single-site precision to excite ions from one spin level to some excited state, and detect the subsequent fluorescence. This allows local $\sigma_{z}^{(i)}$ measurements. Repeated measurements at different $i$ will allow to evaluate correlation functions, e.g. $\left\langle\sigma_{z}^{(i)} \sigma_{z}^{(j)}\right\rangle$, as has been demonstrated, even with temporal resolution, in Refs $[17,18]$.

A key issue is the scalability of spin model quantum simulations. This topic has been discussed in Refs [15, 35], finding only a slow $N^{1 / 3}$ growth of the errors due to spontaneous emission. Nevertheless, clearly outperforming classical computing resources with trapped ions may require new architectures, as the quantum simulation of a two-dimensional system with hundreds of ions in a Penning trap [16].

\section{Dimerization transition}

We have studied by means of exact diagonalization chains of up to 20 ions. The only tunable parameter in our study is the detuning $\delta \equiv \tilde{\omega}_{\alpha}-\omega_{\mathrm{COM}}$, where $\omega_{\mathrm{COM}}$ refers to the frequency of the center-of-mass mode, that is, the transverse mode of largest energy. For convenience, we have chosen ${ }^{171} \mathrm{Yb}^{+}$ions, with equilibirum distance of $10 \mu \mathrm{m}$ at a radial trap frequency $\omega_{\text {trap }}=2 \pi \times 5 \mathrm{MHz}$. As shown in Figure 1(c), (d), for very small detuning, $\delta=1 \mathrm{kHz}$, the system is close to the Lipkin-Meshkov-Glick limit: Any pair of ions interacts with almost the same strength. For large detuning, $\delta \gtrsim 1,000 \mathrm{kHz}$, the interactions decay with $1 / r^{3}$. For intermediate values, the interactions between near neighbors becomes similar to the Haldane-Shastry interactions.

In Figure 2(a), we plot the overlap of the ground state of 16 ions with the ground state manifold of the Majumdar-Ghosh model, and the Haldane-Shastry model as a function of the detuning. In the limit of small detuning, the ground state is almost fully dimerized. Increasing the detuning, the ground state of the Haldane-Shastry model becomes
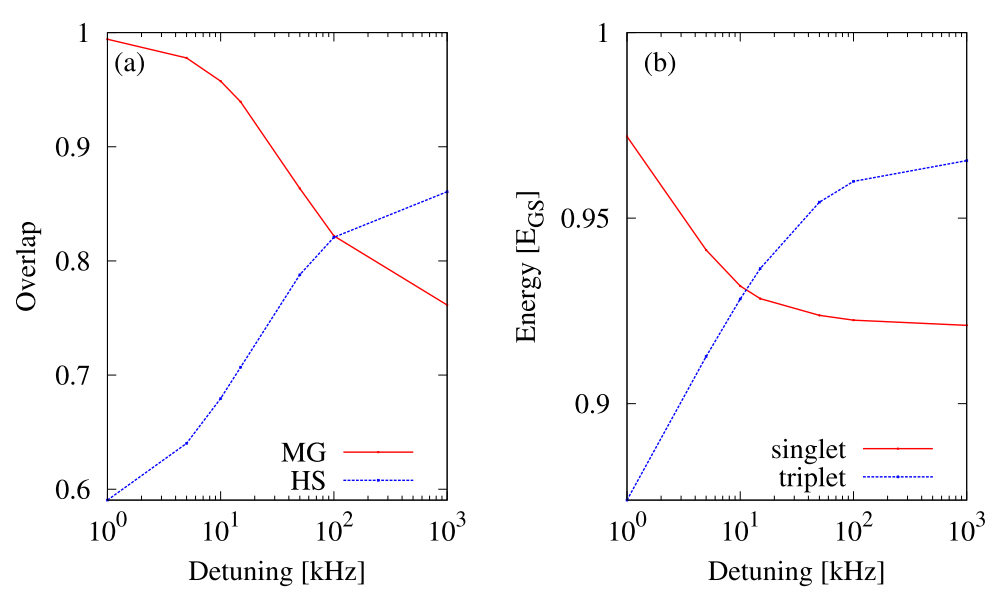

Figure 2 System behavior upon tuning. (a) Overlap of the ground state of $N=16$ ions with the ground state manifold of the Majumdar-Ghosh model, and the Haldane-Shastry model as a function of the detuning. (b) Energies of the first singlet and the first triplet excitation (normalized by the ground state energy), as a function of the detuning. 
more relevant. One has to note, however, that (for 16 particles) this state is far from being orthogonal to the dimerized subspace. In fact, it has itself an overlap of 0.73 with the dimerized manifold.

A sharp criterion for the transition from a dimerized state to a long-range ordered state can be inferred from Figure 2(b), where the energy of the lowest singlet and the lowest triplet excitation is plotted. For the $J_{1}-J_{2}$ model, it is known that the crossing of these energies mark with high precision, even in small systems, the dimerization transition [36]. In our case, the triplet becomes the low-lying excitation for $\delta>10 \mathrm{kHz}$. In this context, we also note that, at $\delta=1 \mathrm{kHz}$, in total 118 singlet states have lower energy than the triplet state. Still, this is far from $C_{16}=1,430$, the number of singlet states providing the ground state manifold of the antiferromagnetic Lipkin-Meshkov-Glick model. But in contrast, at $\delta=5 \mathrm{kHz}$, the lowest triplet state already reaches the 7th position in the energy spectrum.

\subsection{Entanglement entropy}

A dimer is a pair of maximally entangled spins. A measure which localizes the entanglement within a system, and which is thus able to identify dimerization, is the entanglement entropy. This quantity considers bipartitions of the system, and measures the entanglement between the two subsystems. In our case the spins form an one-dimensional array, and it is thus natural to consider the bipartitions $A=\{1, \ldots, \ell\}$ and $B=\{\ell+1, \ldots, N\}$. The entanglement entropy is then a function of $\ell$, defined as

$$
\mathcal{S}(\ell)=-\operatorname{Tr}\left[\hat{\rho}(\ell) \log _{2} \hat{\rho}(\ell)\right]
$$

where $\hat{\rho}(\ell)$ is the density matrix of the subsystem $A$.

The nearest-neighbor dimerized states, $\left|\Psi_{ \pm}\right\rangle$of Eq. (9), are characterized by a strongly alternating behavior of the entanglement entropy, as shown in Figure 3: A cut through every second bond will yield strong entanglement, $\mathcal{S}(\ell)=1$, as the spins $\ell$ and $\ell+1$ are dimerized, whereas on the other bonds no quantum information is shared, $\mathcal{S}(\ell)=0$. Such alternations, however, are not present for the Majumdar-Ghosh dimer with periodic boundary conditions [37]. In that case, both dimer states $\left|\Psi_{+}\right\rangle$and $\left|\Psi_{-}\right\rangle$, will equally

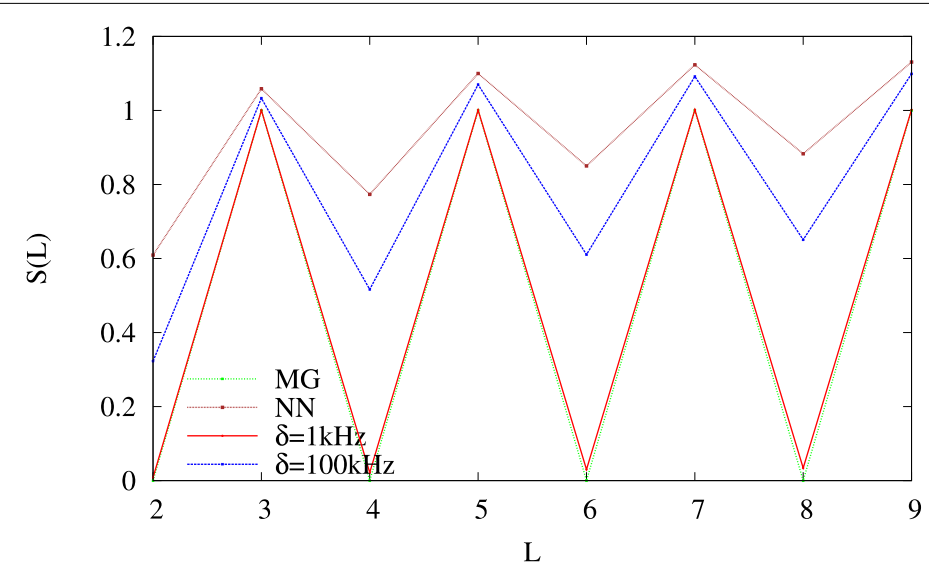

Figure 3 Entanglement entropy. We plot the entanglement entropy of the ionic system with $N=18$ for detuning $\delta=1 \mathrm{kHz}$ and $\delta=100 \mathrm{kHz}$, and for ideal Heisenberg chains with open boundary conditions and nearest-neighbor interactions (NN) and Majumdar-Ghosh (MG) interactions. 
contribute to the ground state manifold, and thus completely wash out the pattern. It has been discussed in Ref. [38] for a broader class of spin models that alternating behavior is characteristic for systems with open boundary conditions, though typically with a much smaller amplitude than in the case of the Majumdar-Ghosh chain.

In Figure 3, we plot the entanglement entropy of different systems with $N=18$ spins: the ionic system with detuning $\delta=1 \mathrm{kHz}$ and $\delta=100 \mathrm{kHz}$, an ideal Heisenberg chain with nearest neighbor interactions, and the Majumdar-Ghosh chain. In all cases, we have applied open boundary conditions, and accordingly we find alternating behavior of the entanglement entropy. As expected, these alternations are strongest for the Majumdar-Ghosh chain, and weakest for the nearest-neighbor Heisenberg model. The entanglement entropy of ionic systems with $\delta=1 \mathrm{kHz}$ behaves very similar to the entanglement entropy of the Majumdar-Ghosh model, proving the dimerized nature of the phase. At $\delta=100 \mathrm{kHz}$, the curve alternates less and comes closer to the entanglement entropy of the nearestneighbor Heisenberg model. This shows that the tendency of nearest neighbors to form singlets is still present, but also more remote spins become entangled.

\subsection{Spin-spin correlations}

An experimentally accessible quantity which nicely displays the different entanglement properties are spin correlations. While correlations will, in principle, depend on the position of the spins, we define an average which depends only on the distance $d$ between the spins:

$$
C(d)=\frac{1}{N-d} \sum_{i=1}^{N-d}\left\langle\sigma_{z}^{(i)} \sigma_{z}^{(i+d)}\right\rangle
$$

To some extent this restores periodic boundary conditions. Furthermore, we perform a finite-size scaling (taking into account all even system sizes from $N=10$ to $N=20$ ).

The results, for $\delta=100 \mathrm{kHz}$, are shown in Figure 4 . The sign of the correlations alternates with odd/even $d$. The correlations compare well with the correlations of the HaldaneShastry model, despite the different boundary conditions. The decay is slightly too fast,
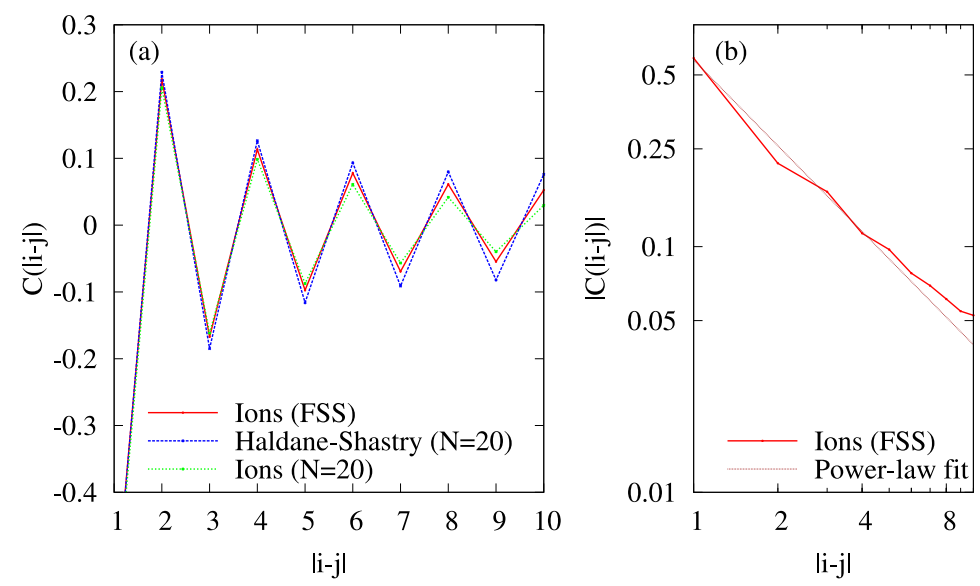

Figure 4 Correlations for large detuning. (a) Correlations of the ionic system ( $\delta=100 \mathrm{kHz}$ ) after finite-size scaling (FSS), and for $N=20$, in comparison with the correlations in the Haldane-Shastry model (with periodic boundary). (b) Power-law fit to the correlations shows a 1/r ${ }^{\alpha}$ decay, with $\alpha=1.15 \pm 0.05$. 


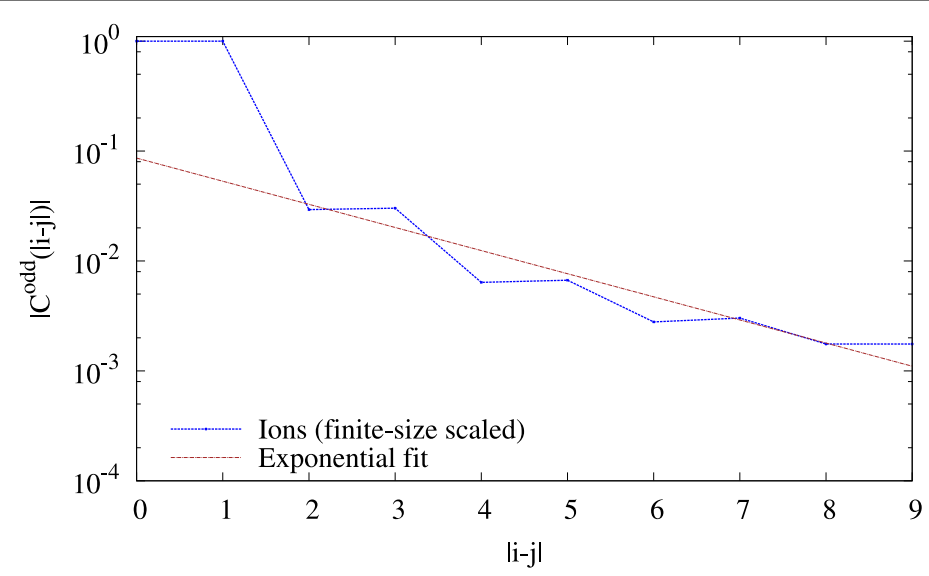

Figure 5 Correlations for small detuning. Staggered correlations (defined in Eq. (20) of the ionic system $(\delta=1 \mathrm{kHz})$ after finite-size scaling (FSS). An exponential fit models well the decay.

but through finite-size scaling a slightly better agreement is obtained. One should also note that for large $d$, comparable to the system size, edge effects are not averaged out by the definition of Eq. (19). The plot in Figure 4(b) demonstrates that the decay can be modeled by a power-law: $C(d) \propto(-1)^{d} r^{-\alpha}$, with $\alpha=1.15 \pm 0.05$.

On the dimerized side, we find correlations as shown in Figure 5. Since $C(d)$ as defined in Eq. (19) would average out the large dimer correlations with the essentially uncorrelated bonds between two dimers, we have defined

$$
C^{\text {odd }}(d)=\frac{1}{i_{\max }} \sum_{i=1}^{i_{\max }}\left\langle\sigma_{z}^{(2 i-1)} \sigma_{z}^{(2 i-1+d)}\right\rangle,
$$

where $i_{\max }=(N-d) / 2$ for $d$ even, and $i_{\max }=(N-d+1) / 2$ for $d$ odd. With such definition, we find that $C^{\text {odd }}(d)$ takes its maximum value $-1 / 4$ for $d=1$, demonstrating the strong anticorrelations between every second nearest-neighbor pair. For larger distances, the value rapidly decreases. The sign alternates for odd/even $d$. As shown in Figure 5, the decay takes place exponentially.

\section{Quench dynamics}

So far we have considered ground state properties of the trapped ion quantum simulation of the Heisenberg model. In two very recent experiments [17, 18], trapped ions have been demonstrated to be particularly useful for studying also the dynamics of interacting spins. This can be done by exciting the system through either a local or a global quench. In the following, we will investigate the system's response to local quenches in both the dimerized and the quasi-long-range ordered regime. Afterwards, we study a global quench through the dimerization transition.

\subsection{Local quenches}

We consider a system which initially is prepared in the ground state of $H$ given by Eq. (17), that is, a system with pure spin-spin interactions. As discussed in the previous section, for sufficiently small detuning, this is a dimerized state, whereas larger detuning yields 


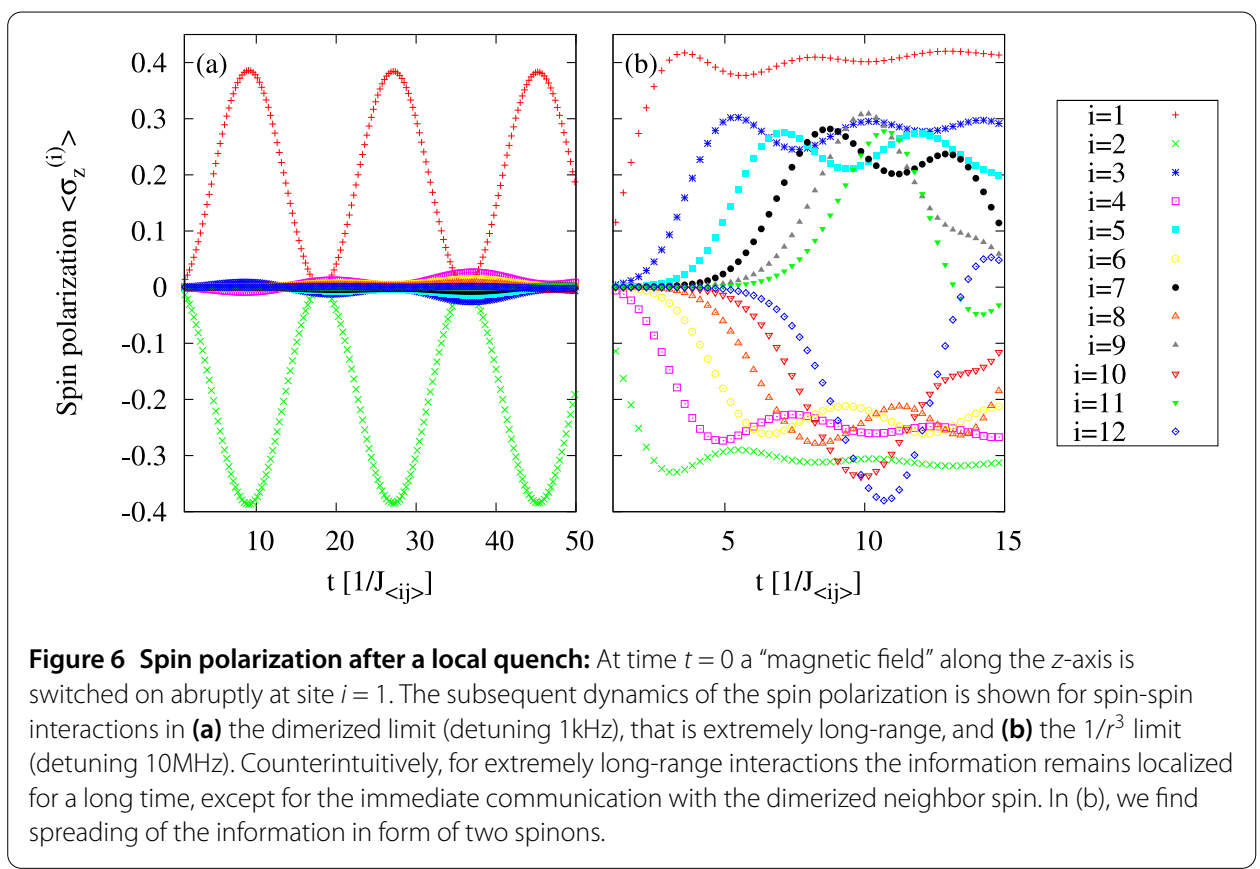

a ground state with algebraically decaying correlations. In both cases, the spin polarization $\left\langle\sigma_{z}^{(i)}\right\rangle$ is zero on all sites $i$. We then create locally a finite spin polarization by abruptly switching on a magnetic field term at one site, for instance at the position of the outermost left spin, $i=1$. Subsequently, we evolve the system under the new Hamiltonian $H^{\prime}=H+B S_{z}^{(1)}$. The evolution of the spin polarization is shown for a system of $N=12$ spins in Figure 6.

In the dimerized system, Figure 6(a), the magnetic field induces a spin oscillation of the first spin. Simultaneously, also the second spin performs an oscillation in the opposite direction. This behavior is an apparent consequence of the strong anticorrelations between the two spins. Interestingly, all other spins remain almost unaffected by the magnetic field for a long time.

A very different behavior is found in the quasi long-range ordered system. Surprisingly, despite the shorter range of interactions, the local perturbation now influences distant spins in significantly less time. Again, the quench on site $i=1$ immediately affects the spin at site $i=2$, as a consequence of strong anticorrelations between neighboring spins. However, as seen in Figure 6(b), the spin polarization also propagates quickly to further spins, leading to positive (negative) spin polarization of the odd (even) spins. Therefore, we may interpret the excitation as a pair of spinons, that is, two spin flips on neighboring sites traveling through the chain. In Figure 7, we further analyze our data by determining the time at which a spin is "activated". Herefore, we demand that $\left|\left\langle\sigma_{z}^{(i)}\right\rangle\right|>0.02$. With this, we are able to define a spinon velocity, plotted in Figure 7(b). On average, this velocity compares well with spinon velocity in the Haldane-Shastry model, $v_{s}=J \frac{\pi}{2}$. At any point in time, the velocities remain below the Lieb-Robinson bound for $S_{z}$ in the nearest-neighbor Heisenberg model, $v_{\mathrm{LR}}=e J[39]$.

An alternative but similar way of performing a local quench is by a projective measurement of one spin. The subsequent dynamics is then described purely by the Hamiltonian $H$, and is found to be qualitatively similar to the dynamics described above. The main 

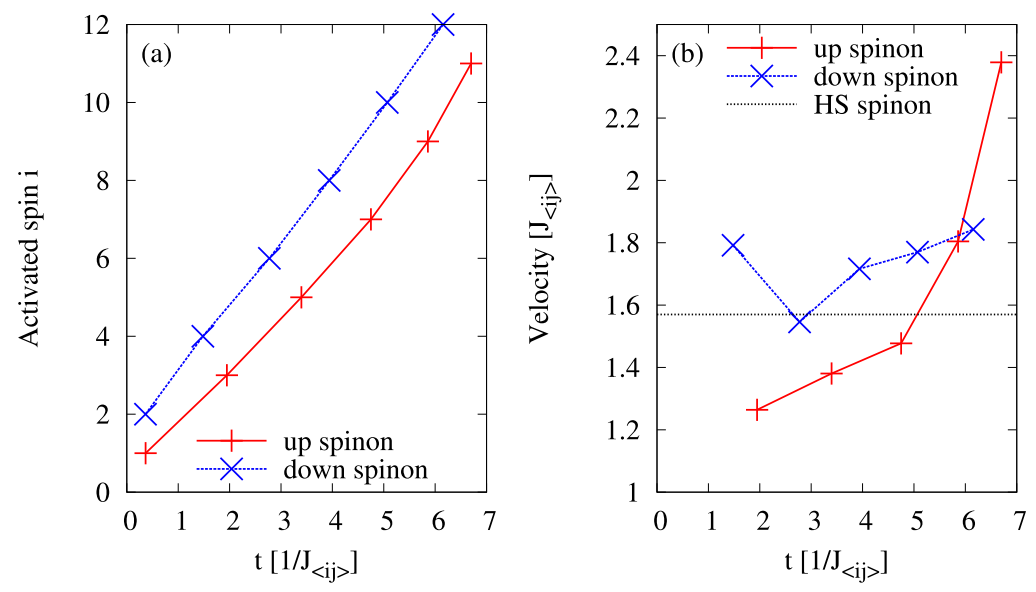

Figure 7 Velocity of excitations. (a) Position vs. time of a spin when it starts being polarized $\left(\left\langle\sigma_{z}^{(i)}\right\rangle>0.02\right)$ in a system with $1 / r^{3}$ decaying interaction (detuning $10 \mathrm{MHz}$ ). (b) Corresponding spinon velocity as a function of time. The black line corresponds to the spinon velocity in the Haldane-Shastry model.

difference is that, in the system with quasi long-range correlations, the projective measurement will immediately affect all spins to a certain extent, obscuring the signal of the spinons.

While in practice it might be difficult to maintain coherence during the whole evolution shown in Figure 6(a), up to times of the order $50 J^{-1}$, the striking differences between Figure $6(\mathrm{a})$ and (b) become apparent already on a much smaller time scale, say $5 J^{-1}$. For typical values of $J$, this is of the order of $10 \mathrm{~ms}$, which is smaller than the duration of measurements in Ref. [18].

\subsection{Global quenches}

We have also studied the systems' dynamics following a global quench. Here an interesting possibility is to abruptly change the range of interactions by changing the detuning. Thereby one can quench through the dimerization transition. Starting from the dimerized ground state, where each spin is correlated only to one neighbor, one can observe the creation of entanglement between more distant spins.

As a first observation we note that the initially dimerized pairs remain strongly anticorrelated for all times. For the correlations between the first and the second spin, we have $C_{12}(t)<-0.8 \forall t$. In Figure 8, we show the growth of correlations between the first spin and the remaining spins, to which no correlations exist in the beginning. In analogy to the behavior of spin polarization discussed before, the spins at odd positions will, in the first place, become correlated with the first spin, while the even spins are getting anticorrelated. As a guide to the eye, the vertical lines in Figure 8 mark the "trajectory" of a signal traveling with the Haldane-Shastry spinon velocity, $v_{s}=\pi / 2$. The first line corresponds to a traveled distance of one unit of length. For subsequent lines, the distance is increased by two. As the first and the second ions are dimerized, we may always consider the distance between an ion $i$ to the second ion, $i=2$. We then find that the velocity with which correlations reach the spins at $i=3, i=5$, and $i=7$ coincides well with the spinon velocity. Interestingly, the anticorrelations with the even spins are transmitted more rapidly. A remarkable increase of velocity is found towards the end of the chain: Spins at $i=7, i=9$, and $i=11$ 


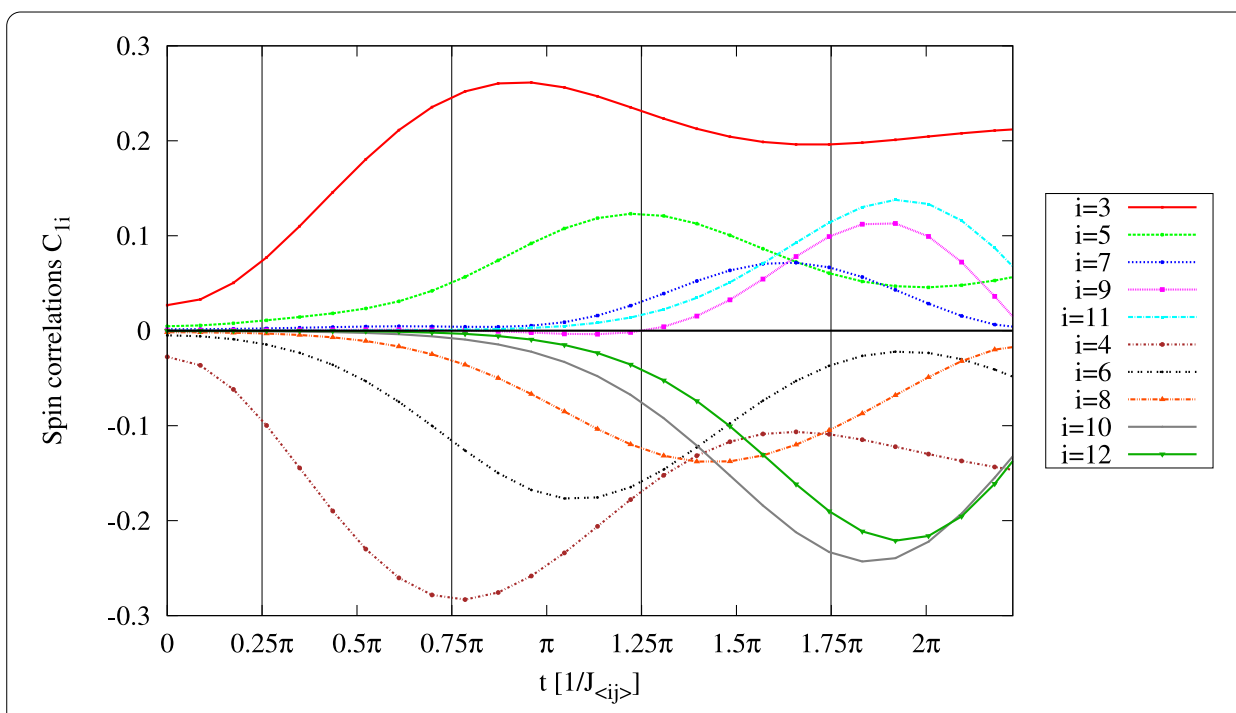

Figure 8 Spin correlations after a global quench: At time $t=0$, the system is quenched by changing the detuning from $1 \mathrm{kHz}$ to $10 \mathrm{MHz}$, i.e., the range of interactions is changed. Before the quench, the system is in a dimerized state of localized correlations, after the quench correlations spread throughout the whole system.

become correlated with the first spin almost simultaneously. Even more surprisingly, the correlation with the spin at $i=11$ starts to grow slightly earlier than the correlation with the spin at $i=9$. An explanation of this behavior could be the fact that in the moment of the global quench excitations are created at all positions. Each excitation will, in the first place, build up correlations between neighboring spins, but nonlocality of correlations can provide an immediate "bridge" between spins at a larger distance.

\section{Nonlocality witness}

In the previous sections, we have discussed one intriguing aspect of quantum mechanics which can be studied in the ionic system: entanglement. A strongly related and particular striking feature of entanglement is the nonlocality of correlations [40]. Nonlocality can be defined as the impossibility of classically simulating the outcome of a local measurement while a second, remote measurement is performed unless some information obtained during this measurement is shared. As first shown by JS Bell, nonlocality is witnessed by the violation of some inequalities which local correlations have to fulfill [41]. In general, unfortunately, Bell inequalities depend on various high-order correlation functions, and it is thus difficult to witness nonlocality in a system. In Ref. [27], however, it has been proposed to detect nonlocality by measurement of two-body correlators only. As a specific example, a Bell inequality has been given which is violated by the spin-polarized Dicke state $\left|D_{N / 2, N / 2}\right\rangle$. It reads

$$
\frac{N(N-1)}{4} S_{00}+\frac{N}{2} S_{10}-\frac{1}{2} S_{11}+\frac{1}{4} N(N-1)(N+2) \geq 0 .
$$

Here, $S_{i j} \equiv \sum_{k \neq l}\left\langle\hat{m}_{i}^{(k)} \hat{m}_{j}^{(l)}\right\rangle$ are the correlations of two measurements $\hat{m}$ taken at sites $k$ and $l$. On each site, one may take $\hat{m}_{0} \equiv \sigma_{z}$ and $\hat{m}_{1} \equiv \cos \theta \sigma_{z}+\sin \theta \sigma_{x}$. If a $\theta$ for which inequality (21) is violated exists, the system must have non-local correlations. 
While inequality (21) turns out to be unable to detect nonlocality on the antiferromagnetic side of the ionic setup, a chance for violation exists on its ferromagnetic side. For sufficiently small detuning, the sytem is in the Lipkin-Meshkov-Glick limit of spatially independent interactions, and for $J<0$, states with maximal spin are ground state of the Hamiltonian $H \approx J\left(\mathbf{S}^{2}-\frac{3}{4} N\right)$. The ground state manifold is thus given by all $N+1$ Dicke states. To lift the degeneracy, one could apply a magnetic field: A field in $z$-direction yields either $D_{0, N}$ or $D_{N, 0}$ as the unique ground state. The state $\left|D_{N / 2, N / 2}\right\rangle$ becomes unique ground state if a staggered magnetic field along $z$-direction is applied. Alternatively, even without applying a symmetry-breaking field, the degeneracy is lifted in favor of $\left|D_{N / 2, N / 2}\right\rangle$ by switching off the interactions $J_{z}^{(i, j)} \rightarrow 0$. The system is then govered by $H_{\mathrm{LMG}}$ given in Eq. (12).

In these cases, non-locality can be proven via inequality (21), as the spin-spin correlation of $\left|D_{N / 2, N / 2}\right\rangle$ are given by

$$
\begin{aligned}
& S_{x x} \equiv \sum_{i \neq j}\left\langle\sigma_{x}^{(i)} \sigma_{x}^{(j)}\right\rangle=\frac{N^{2}}{2}, \\
& S_{z z} \equiv \sum_{i \neq j}\left\langle\sigma_{z}^{(i)} \sigma_{z}^{(j)}\right\rangle=-N, \\
& S_{z x} \equiv \sum_{i \neq j}\left\langle\sigma_{z}^{(i)} \sigma_{x}^{(j)}\right\rangle=0 .
\end{aligned}
$$

With this, inequality (21) is violated. To assert the feasibility of such non-locality witness in the ionic system, we have calculated the ground state of a system of 14 ions, with $J_{z}^{(i, j)}=0$, while $J_{x}^{(i, j)}$ and $J_{y}^{(i, j)}$ given by Eq. (16). Indeed, the system is found in the Dicke state $\left|D_{N / 2, N / 2}\right\rangle$ with fidelity $>0.99$ for $|\delta|<1 \mathrm{kHz}$. By increasing the absolute value of the negative detuning, we find that, while $S_{z z}$ and $S_{z x}$ remain constant, $S_{x x}$ decreases. Thus, for $S_{x x}$ below a critical value $S_{x x}^{\text {crit }}=N(N-2) / 2$, inequality (21) will not be violated anymore. For $N=14$, we numerically found $S_{x x}=89.6>S_{x x}^{\text {crit }}$ at $\delta=-2.8 \mathrm{kHz}$, while $S_{x x}=73.3<S_{x x}^{\text {crit }}$ at $\delta=-3.0 \mathrm{kHz}$. In the same parameter range, the overlap of the ground state with the Dicke state drops suddenly: While it remains above 0.95 up to $|\delta|=2.5 \mathrm{kHz}$, it reaches zero for $|\delta|=3.2 \mathrm{kHz}$.

In this regime of relatively large detuning, other phonons than the center-of-mass mode strongly affect the spin-spin interactions. This gives rise $t$ very peculiar interaction patterns consisting of attractively and repulsively interacting pairs. It would, however, be striking if non-locality of correlations could also be witnessed in a system where interactions are short-ranged. Interestingly, we find that the short-range ferromagnetic Heisenberg chain behaves exactly the same way as the long-range chain does: $N+1$ Dicke states form a ground state manifold in which degeneracies can be lifted by magnetic fields, in particular, in favor of $\left|D_{N / 2, N / 2}\right\rangle$ by a staggered magnetic field. Certainly, the ionic setup discussed here does not immediately allow for implementation of the ferromagnetic shortranged Heisenberg model due to the mentioned mixing of different phonon modes, but it could be achieved by switching from transverse to longitudinal phonons as transmitter of interactions. In that case, the center-of-mass mode is lowest in energy, and the negative detuning will not interfere with other modes. Moreover, additional control could be implemented through additional Raman couplings [34]. Alternatively, also atoms in optical waveguides or phononic crystals allow for implementing spin models with controllable interactions, and could be tuned into a ferromagnetic short-range regime [42]. We also note 
that by using adiabatic passage techniques the Dicke state $\left|D_{N / 2, N / 2}\right\rangle$ has recently been produced in trapped ion systems [43].

\section{Summary}

We have simulated a quantum simulation of the Heisenberg model with trapped ions. In particular, we have investigated the influence of a single control parameter, the detuning, on the system. This parameter allows to tune the range of the interactions. We have shown that this can trigger a dimerization transition. To demonstrate dimerization, we have calculated the entanglement entropy and two-spin correlation functions in systems of up to $N=20$ ions. Studying the response to local quenches, we were able to distinguish between the two regimes also by the system's dynamics: For long times, local excitations do not spread in the dimerized system. In contrast to this, they are carried through the whole chain in form of spinons in the regime with quasi-long-range order. We also have calculated the spreading of correlations following a global quench, and found some signature of non-locality. Apart from this, we have explored the possibility of witnessing non-locality by measurement of two-spin correlation functions on the ferromagnetic side of the model. In summary, the trapped-ion quantum simulation is able to test basic foundations of quantum mechanics, and to study both ground state properties and dynamics of complicated, long-ranged models.

\section{Appendix 1: Photon-phonon interaction}

The key ingredient to achieve spin-spin interactions are Raman couplings between the two levels. The Hamiltonian of the system then reads $H=H_{0}+\sum_{\alpha=x, y, z} h_{\alpha}$, where $H_{0}$ contains the electronic and the motional energy of the ions,

$$
H_{0}=\sum_{i=1}^{N} \frac{\hbar \omega_{0}}{2} \sigma_{z}^{(i)}+\sum_{m=1}^{3 N}\left(\hat{n}_{m}+\frac{1}{2}\right),
$$

and $h_{\alpha}$ describes the interactions with the photons,

$$
h_{\alpha}=\sum_{i=1}^{N} \hbar \Omega_{\alpha}\left(\mathrm{e}^{i\left(\mathbf{k}_{\alpha} \cdot \mathbf{r}^{(i)}-\omega_{\alpha}+\varphi_{\alpha}\right)}+\text { H.c. }\right) \tau_{\alpha}^{(i)} \text {. }
$$

Here, $\hbar \omega_{0}$ is the energy difference between the two internal levels, $\sigma_{z}$ is a Pauli matrix. The phonons in the system are counted by $\hat{n}_{m}=\hat{a}_{m}^{\dagger} \hat{a}_{m}$, for each mode denoted by $m$. The different Raman couplings, denoted by the index $\alpha$, give rise to Rabi frequencies $\Omega_{\alpha}$. The fields have wave vector $\mathbf{k}_{\alpha}$, frequency $\omega_{\alpha}$, and a phase $\varphi_{\alpha}$. The position of the ions is denoted by $\mathbf{r}^{(i)}$, and the action of the field on the internal state of each ion is expressed by $\tau_{\alpha}^{(i)}$. We choose the polarization of the couplings such that $\tau_{x}^{(i)}=\tau_{y}^{(i)} \equiv \sigma_{x}^{(i)}$, whereas $\tau_{z}^{(i)} \equiv \sigma_{z}^{(i)}$.

Several assumptions on the Hamiltonian parameters can be made to simplify the expression. First of all, in the Lamb-Dick regime we have $\mathbf{k}_{\alpha} \cdot \mathbf{r}^{(i)} \ll 1$, and we therefore approximate $\mathrm{e}^{i\left(\mathbf{k}_{\alpha} \cdot \mathbf{r}^{(i)}\right)} \approx 1+i \mathbf{k}_{\alpha} \cdot \mathbf{r}^{(i)}=1+\sum_{m} \eta_{m \alpha}^{(i)}\left(\hat{a}_{m}+\hat{a}_{m}\right)$. In the latter step, we have replaced the ions' coordinates $\mathbf{r}^{(i)}$ by the normal modes around their equilibrium position. The scalar product $\mathbf{k}_{\alpha} \cdot \mathbf{r}^{(i)}$ is then rewritten in terms of Lamb-Dicke parameters $\eta_{m \alpha}$. These parameters measure the strength of the couplings between the photons with wave vector $\mathbf{k}_{\alpha}$ and the phononic mode $m$. 
To obtain the values of the Lamb-Dicke parameters, we have to calculate the normal modes in direction of $\mathbf{k}_{\alpha}$, which may be either transverse to the ion chain in $x$ - or $y$ direction, or longitudinal along the $z$-axis. We obtain the normal modes, denoted by the $N \times N$ matrix $\mathcal{M}_{m, i}^{\alpha}$, by diagonalizing the vibrational Hamiltonian $\mathcal{K}^{\alpha}$,

$$
\mathcal{M}_{m, i}^{\alpha} \mathcal{K}_{m m^{\prime}}^{\alpha} \mathcal{M}_{m^{\prime}, i^{\prime}}^{\alpha}=\omega_{m \alpha}^{2} \delta_{i, i^{\prime}}
$$

Here, we label the phononic modes by $m \in 1, \ldots, N$ and an additional label $\alpha \in\{\mathrm{x}, \mathrm{y}, \mathrm{z}\}$ specifying the direction of the modes. Having solved Eq. (27), the Lamb-Dicke parameters are expressed by $\eta_{m \alpha}^{(i)}=\sqrt{\frac{\hbar}{2 M \omega_{m \alpha}}} \mathcal{M}_{m \alpha}$, with $M$ the ion's mass.

The kernel $\mathcal{K}^{\alpha}$ contains the Coulomb repulsion and the external trapping of frequency $\omega_{\text {trap }, \alpha}$ along each direction. Assuming linearly arranged and equidistant equilibrium positions, it reads:

$$
\begin{aligned}
\mathcal{K}_{m, m^{\prime}}^{\alpha}= & \delta_{m, m^{\prime}} c_{\alpha}\left[\frac{e^{2} / M}{4 \pi \epsilon_{0}\left|\mathbf{r}^{(m)}-\mathbf{r}^{\left(m^{\prime \prime}\right)}\right|^{3}}\right] \\
& +\left(1-\delta_{m, m^{\prime}}\right)\left[\omega_{\text {trap }, \alpha}^{2}-c_{\alpha} \sum_{m^{\prime \prime}(\neq m)} \frac{e^{2} / M}{4 \pi \epsilon_{0}\left|\mathbf{r}^{(m)}-\mathbf{r}^{\left(m^{\prime \prime}\right)}\right|^{3}}\right]
\end{aligned}
$$

where $c_{x, y}=1, c_{z}=-2, e$ the unit of charge, and $\epsilon_{0}$ the electric constant. For a simulation of the Haldane-Shastry model, it turns out to be convenient to generate all interactions using transverse mode. Assuming isotropy in the transverse directions, we will be able to suppress the index $\alpha$ in the following.

It is convenient to transform the $h_{\alpha}$ into the interaction picture of $H_{0}: h_{\alpha} \rightarrow \mathrm{e}^{i H_{0} t / \hbar} h_{\alpha} \times$ $\mathrm{e}^{i H_{0} t / \hbar}$. This amounts for replacing $\hat{a}_{m} \rightarrow a_{m} \mathrm{e}^{i \omega_{m} t}$ and $\sigma_{x}=\frac{1}{2}\left(\mathrm{e}^{i \omega_{0} t} \sigma_{+}\right)+$H.c.. Finally, we make a rotating-wave approximation, that is, we neglect all fast oscillating terms in the Hamiltonian. To do this, we have to make some assumptions about the energies involved in the Hamiltonian: We tune the frequencies $\omega_{x}$ and $\omega_{y}$, that is, the frequencies of the light field in $h_{x}$ and $h_{y}$ of Eq. (26), close to $\omega_{0}+\omega_{m}$, that is, the frequency of a spin flip and the creation of a phononic mode. With this choice we obtain:

$$
\begin{aligned}
& h_{x}=\frac{\hbar}{2} \Omega_{x} \sum_{i=1}^{N} \sum_{m} \eta_{m}^{(i)}\left(\hat{a}_{m} \mathrm{e}^{-i\left(\omega_{x}-\omega_{0}-\omega_{m}\right) t}+\text { H.c. }\right) \sigma_{x}^{(i)}, \\
& h_{y}=\frac{\hbar}{2} \Omega_{y} \sum_{i=1}^{N} \sum_{m} \eta_{m}^{(i)}\left(\hat{a}_{m} \mathrm{e}^{-i\left(\omega_{y}-\omega_{0}-\omega_{m}\right) t}+\text { H.c. }\right) \sigma_{y}^{(i)} .
\end{aligned}
$$

Here, we have set the phase of the $k_{x}$-laser field, $\phi_{x}$, set to zero. For the coupling in $y$ direction, we choose this phase to be $\phi_{y}=\pi$, which effectively replaces $\sigma_{x}$ by the $\sigma_{y}$ matrix. Note that in the rotating-wave approximation, we have also neglected terms oscillating with $\omega_{\alpha}-\omega_{0}+\omega_{m}$, which is justified if we tune $\omega_{\alpha}-\omega_{0}$ towards the upper edge of the phonon spectrum.

Since no spin flip should be associated to the $h_{z}$ coupling, the corresponding frequency $\omega_{z}$ must be tuned close to $\omega_{m}$. In the rotating-wave rotation, the corresponding Hamiltonian reads

$$
h_{z}=i \hbar \Omega_{z} \sum_{i=1}^{N} \sum_{m} \eta_{m}^{(i)}\left(\hat{a}_{m} \mathrm{e}^{-i\left(\omega_{z}-\omega_{m}\right) t}+\text { H.c. }\right) \sigma_{z}^{(i)} .
$$




\section{Appendix 2: Time evolution}

The time evolution of a system with a time-dependent Hamiltonian $H(t)$ can be calculated by applying the Magnus formula [44],

$$
U(t, 0)=\exp \left[-\frac{i}{\hbar} \int_{0}^{t} \mathrm{~d} t^{\prime} H\left(t^{\prime}\right)-\frac{1}{2 \hbar^{2}} \int_{0}^{t} \mathrm{~d} t^{\prime} \int_{0}^{t^{\prime}} \mathrm{d} t^{\prime \prime}\left[H\left(t^{\prime}\right), H\left(t^{\prime \prime}\right)\right]\right] .
$$

For a single coupling $\tau_{\alpha}$, this yields

$$
U_{\alpha}(t, 0)=\exp \left[\sum_{i} \varphi_{\alpha}^{(i)}(t) \tau_{\alpha}^{(i)}-\sum_{i, j} \xi_{\alpha}^{(i, j)}(t) \tau_{\alpha}^{(i)} \tau_{\alpha}^{(j)}\right],
$$

where $\varphi_{\alpha}^{(i)}=\sum_{m}\left(c_{m \alpha}^{(i)}(t) a_{m \alpha}^{\dagger}-\right.$ H.c. $)$ contains a residual spin-phonon coupling, while the second term describes a spin-spin coupling. As argued in Ref. [14], for sufficiently large detuning from the motional sideband, all oscillatory terms in $c_{m \alpha}^{(i)}(t)$ and $\xi_{\alpha}^{(i, j)}(t)$ can be neglected, and the long-term time evolution is dominated by a single term in $\xi_{\alpha}^{(i, j)}(t)$ which is linear in $t$. Thus, we can set $c_{m \alpha}^{(i)} \approx 0$, and $\xi_{\alpha}^{(i, j)}(t) \approx i J_{\alpha}^{(i, j)} t$, with

$$
J_{\alpha}^{(i, j)}=\Omega_{\alpha}^{2} \sum_{m} \frac{\eta_{m \alpha}^{(i)} \eta_{m \alpha}^{(j)}}{4\left(\omega_{\alpha}-\omega_{0}-\omega_{m}\right)} .
$$

The time evolution is thus identical to the one of a spin model with spin-spin coupling $J_{\alpha}^{(i, j)}$.

In the presence of more than one couplings, since $\left[h_{\alpha}, h_{\beta}\right] \neq 0$, the time evolution is not simply the product of all $U_{\alpha}$, but consists of additional terms. As in Ref. [12, 20], there are terms which stem from the non-commutativity of the spin matrices. Since all three couplings shall be transmitted by transverse phonons, for at least one pair of couplings also the phononic part will interfere. We get $U \simeq\left(\prod_{\alpha} U_{\alpha}\right)\left(\prod_{\alpha \neq \beta} U_{\alpha \beta}\right)$ with

$$
\left.U_{\alpha \beta}(t, 0)=\exp \left\{-\frac{\Omega_{\alpha} \Omega_{\beta}}{2 \hbar^{2}} \delta_{i j} \chi_{\alpha \beta}^{(i)}(t)\left[\sigma_{\alpha}^{(i)}, \sigma_{\beta}^{(i)}\right]+\varrho_{\alpha \beta}(t) \sigma_{\beta}^{(j)} \sigma_{\alpha}^{(i)}\right)\right\}
$$

The functions $\chi_{\alpha \beta}^{(i)}$ are given by the integral

$$
\chi_{\alpha \beta}^{(i)}(t)=\sum_{m, n} \int_{0}^{t} \mathrm{~d} t_{1} \int_{0}^{t_{1}} \mathrm{~d} t_{2}\left(a_{m} e^{-i \tilde{\omega}_{m \alpha} t_{1}}+\text { H.c. }\right)\left(a_{n} e^{-i \tilde{\omega}_{n \beta} t_{2}}+\text { H.c. }\right)
$$

with $\tilde{\omega}_{m \alpha} \equiv \omega_{\alpha}-\omega_{m}$ if $\alpha=\mathrm{z}$, or $\tilde{\omega}_{\alpha} \equiv \omega_{\alpha}-\omega_{0}-\omega_{m}$ if $\alpha=\mathrm{x}$, y. An analog definition holds for $\tilde{\omega}_{n \beta}$. The function $\varrho_{\alpha \beta}(t)$ is given by the integral

$$
\varrho_{\alpha \beta}(t)=\sum_{m} \int_{0}^{t} \mathrm{~d} t_{1} \int_{0}^{t_{1}} \mathrm{~d} t_{2} \cos \left[\tilde{\omega}_{m \alpha} t_{1}-\omega_{m \beta} t_{2}\right] .
$$

Unless $\omega_{m \alpha}=\omega_{n \beta}$, these functions only yield oscillatory terms, and can then be neglected. In that case, the time evolution of the system is equivalent to the one of a spin system with Hamiltonian

$$
H=\sum_{\alpha} \sum_{i \leq j}=J_{\alpha}^{(i, j)} \sigma_{\alpha}^{(i)} \sigma_{\alpha}^{(j)}
$$




\section{Competing interests}

The authors declare that they have no competing interests.

\section{Authors' contributions}

All authors contributed equally to the writing of this paper. All authors read and approved the final manuscript.

\section{Author details}

${ }^{1}$ ICFO-Institut de Ciències Fotòniques, Av. Carl Friedrich Gauss, 3, 08860 Castelldefels, Barcelona, Spain. ${ }^{2}$ ICREA-Institució Catalana de Recerca i Estudis Avançats, Barcelona, 08010, Spain

\section{Acknowledgements}

The authors thank Remigiusz Augusiak and Ravindra Chhajlany for discussions. This work has been supported by EU (SIQS, FP7/2007-2013 Grant No. 323714 EQUAM), ERC (QUAGATUA, OSYRIS), Spanish MINCIN (FIS2008-00784 TOQATA), Generalitat de Catalunya (2009-SGR1289), Alexander von Humboldt Stiftung, and Fundació Cellex.

Received: 26 February 2014 Accepted: 12 May 2014 Published: 3 June 2014

\section{References}

1. Auerbach A: Interacting Electrons and Quantum Magnetism. Berlin: Springer; 1994.

2. Lewenstein M, Sanpera A, Ahufinger V: Ultracold Atoms in Optical Lattices - Simulating Quantum Many-Body Systems. Oxford: Oxford University Press; 2012.

3. Romero-Isart O, Eckert K, Rodó C, Sanpera A: Transport and entanglement generation in the Bose-Hubbard model. J Phys A, Math Theor 2007, 40(28):8019.

4. Hauke P, Tagliacozzo L: Spread of correlations in long-range interacting quantum systems. Phys Rev Lett 2013, 111:207202

5. Schachenmayer J, Lanyon BP, Roos CF, Daley AJ: Entanglement growth in quench dynamics with variable range interactions. Phys Rev X 2013, 3:031015.

6. Lamata L, León J, Schätz T, Solano E: Dirac equation and quantum relativistic effects in a single trapped ion. Phys Rev Lett 2007, 98:253005.

7. Gerritsma R, Kirchmair G, Zähringer F, Solano E: Quantum simulation of the Dirac equation. Nature 2010, 463:68.

8. Schmied R, Wesenberg JH, Leibfried D: New J Phys 2011, 13:115011.

9. Casanova J, Mezzacapo A, Lamata L, Solano E: Quantum simulation of interacting fermion lattice models in trapped ions. Phys Rev Lett 2012, 108:190502.

10. Pons M, Ahufinger V, Wunderlich C, Sanpera A, Braungardt S, Sen(De) A, Sen U, Lewenstein M: Trapped ion chain as a neural network: error resistant quantum computation. Phys Rev Lett 2007, 98:023003.

11. Mintert F, Wunderlich C: Ion-trap quantum logic using long-wavelength radiation. Phys Rev Lett 2001, 87:257904.

12. Porras D, Cirac Jl: Effective quantum spin systems with trapped ions. Phys Rev Lett 2004, 92:207901.

13. Friedenauer A, Schmitz H, Glueckert JT, Porras D, Schaetz T: Simulating a quantum magnet with trapped ions. Nat Phys 2008, 4(10):757-761.

14. Kim K, Chang M-S, Islam R, Korenblit S, Duan L-M, Monroe C: Entanglement and tunable spin-spin couplings between trapped ions using multiple transverse modes. Phys Rev Lett 2009, 103:120502.

15. Kim K, Chang MS, Korenblit S, Islam R, Edwards EE, Freericks JK, Lin GD, Duan LM, Monroe C: Quantum simulation of frustrated Ising spins with trapped ions. Nature 2010, 465:590-593.

16. Britton JW, Sawyer BC, Keith AC, Wang C-CJ, Freericks JK, Uys H, Biercuk MJ, Bollinger JJ: Engineered two-dimensional Ising interactions in a trapped-ion quantum simulator with hundreds of spins. Nature 2012, 484(7395):489-492.

17. Richerme P, Gong Z-X, Lee A, Senko C, Smith J, Foss-Feig M, Michalakis S, Gorshkov AV, Monroe C: arXiv:1401.5088; 2014

18. Jurcevic P, Lanyon BP, Hauke P, Hempel C, Zoller P, Blatt R, Roos CF: arXiv:1401.5387; 2014

19. Deng X-L, Porras D, Cirac Jl: Effective spin quantum phases in systems of trapped ions. Phys Rev A 2005, $72: 063407$.

20. Graß T, Juliá-Díaz B, Kuś M, Lewenstein M: Quantum chaos in su(3) models with trapped ions. Phys Rev Lett 2013, $111: 090404$

21. Koffel T, Lewenstein M, Tagliacozzo L: Entanglement entropy for the long-range Ising chain in a transverse field. Phys Rev Lett 2012, 109:267203.

22. Haldane FDM: Exact Jastrow-Gutzwiller resonating-valence-bond ground state of the spin-(1/2 antiferromagnetic Heisenberg chain with 1/. ${ }^{2}$ exchange. Phys Rev Lett 1988, 60:635-638.

23. Shastry BS: Exact solution of an $S=1 / 2$ Heisenberg antiferromagnetic chain with long-ranged interactions. Phys Rev Lett 1988, 60:639-642.

24. Greiter M: Mapping of Parent Hamiltonians: from Abelian and Non-Abelian Quantum Hall States to Exact Models of Critical Spin Chains. Berlin: Springer; 2011.

25. Majumdar CK, Ghosh DK: J Math Phys 1969, 10:1388.

26. Lipkin H, Meshkov N, Glick AJ: Nucl Phys 1965, 62:188.

27. Tura J, Augusiak R, Sainz AB, Vértesi T, Lewenstein M, Acín A: arXiv:1306.6860; 2013

28. Laughlin RB: Anomalous quantum hall effect: an incompressible quantum fluid with fractionally charged excitations. Phys Rev Lett 1983, 50:1395.

29. Gebhard F, Vollhardt D: Correlation functions for Hubbard-type models: the exact results for the Gutzwiller wave function in one dimension. Phys Rev Lett 1987, 59:1472-1475.

30. Affleck l: Critical behavior of two-dimensional systems with continuous symmetries. Phys Rev Lett 1985, 55:1355-1358

31. Okamoto K, Nomura K: Fluid-dimer critical point in $\mathbf{s}=\mathbf{1 2}$ antiferromagnetic Heisenberg chain with next nearest neighbor interactions. Phys Lett A 1992, 169(6):433-437.

32. Eggert S: Numerical evidence for multiplicative logarithmic corrections from marginal operators. Phys Rev $B 1996$ 54:9612-9615. 
33. Schneider C, Porras D, Schaetz T: Experimental quantum simulations of many-body physics with trapped ions. Rep Prog Phys 2012, 75(2):024401.

34. Korenblit S, Kafri D, Campbell WC, Islam R, Edwards EE, Gong Z-X, Lin G-D, Duan L-M, Kim J, Kim K, Monroe C: Quantum simulation of spin models on an arbitrary lattice with trapped ions. New J Phys 2012, 14(9):095024.

35. Kim K, Korenblit S, Islam R, Edwards EE, Chang M-S, Noh C, Carmichael H, Lin G-D, Duan L-M, Wang CCJ, Freericks JK, Monroe C: Quantum simulation of the transverse Ising model with trapped ions. New J Phys 2011, 13(10):105003.

36. Sandvik AW: Ground states of a frustrated quantum spin chain with long-range interactions. Phys Rev Lett 2010, 104:137204

37. Chhajlany RW, Tomczak P, Wójcik A, Richter J: Entanglement in the Majumdar-Ghosh model. Phys Rev A 2007, 75:032340

38. Laflorencie N, Sørensen ES, Chang M-S, Affleck I: Boundary effects in the critical scaling of entanglement entropy in 1d systems. Phys Rev Lett 2006, 96:100603.

39. Them K: arXiv:1308.2882; 2013.

40. Brunner N, Cavalcanti D, Pironio S, Scarani V, Wehner S: Bell nonlocality. Rev. Mod. Phys. 2014, 86:419.

41. Bell JS: Physics 1964, 1:195.

42. Douglas JS, Habibian H, Gorshkov AV, Kimble HJ, Chang DE: arXiv:1312.2435; 2013.

43. Noguchi A, Toyoda K, Urabe S: Generation of Dicke states with phonon-mediated multilevel stimulated Raman adiabatic passage. Phys Rev Lett 2012, 109:260502.

44. Blanes S, Casas F, Oteo JA, Ros J: The magnus expansion and some of its applications. Phys Rep 2009, 470(5-6):151-238.

doi:10.1140/epjqt8

Cite this article as: Graß and Lewenstein: Trapped-ion quantum simulation of tunable-range Heisenberg chains. EPJ Quantum Technology 2014 1:8.

\section{Submit your manuscript to a SpringerOpen ${ }^{\circ}$ journal and benefit from:}

- Convenient online submission

- Rigorous peer review

- Immediate publication on acceptance

Open access: articles freely available online

- High visibility within the field

- Retaining the copyright to your article 